Ligt, K.M. de, Heins, M., Verloop, J., Smorenburg, C.H., Korevaar, J.C., Siesling, S. Patient-reported health problems and healthcare use after treatment for early-stage breast cancer The Breast: 2019, 46, 4-11

$\begin{array}{lll}\text { Postprint version } & : & 1.0 \\ \text { Journal website } & : & \frac{\text { https://www.thebreastonline.com/article/S0960-9776(19)30479- }}{5 / \text { abstract }} \\ \text { Pubmed link } & : & \underline{\text { https://www.ncbi.nlm.nih.gov/pubmed/30981032 }} \\ \text { DOI } & : \underline{10.1016 / j . b r e a s t .2019 .03 .010}\end{array}$

This is a Nivel certified Post Print, more info at nivel.nl

\title{
Patient-reported health problems and healthcare use after treatment for early-stage breast cancer
}

\author{
K.M. de Ligt ${ }^{\text {a, b, }{ }^{*}, \text { M. Heins }}{ }^{c}$, J. Verloop ${ }^{a}$, C.H. Smorenburg ${ }^{\text {d }}$, J.C. Korevaar ${ }^{c}$, \\ S. Siesling ${ }^{a, b}$
}

a Department of Research, Netherlands Comprehensive Cancer Organisation (IKNL), Utrecht, the Netherlands

b Department of Health Technology and Services Research, Faculty of Behavioural, Management and Social Sciences, Technical Medical Centre, University of

Twente, Enschede, the Netherlands

c NIVEL Netherlands Institute for Health Services Research, Utrecht, the Netherlands

d Department of Medical Oncology, Netherlands Cancer Institute - Antoni van Leeuwenhoek, Amsterdam, the Netherlands

\footnotetext{
a bstract

Background: A clear picture of treatment-related health problems following breast cancer treatment is

useful in anticipating the informational and other needs of patients during follow-up. This study aimed

to identify treatment-related health problems in breast cancer patients up to five years after diagnosis.

Secondly, the use of care associated with these health problems was identified. Methods: 876 surgically-treated female patients diagnosed between 2012 and 2016 with early-stage

breast cancer were asked to complete an online survey about their current health problems and use

of care. Multivariate logistic regression analyses were applied to determine the effect of patient and treatment characteristics on health problems.

Results: 404 patients responded (46\%). The median age was 62.0 years (SD:10.9). Apart from breast surgery, patients had been treated with radiotherapy (72\%), chemotherapy (49\%), antihormonal therapy
} 
Ligt, K.M. de, Heins, M., Verloop, J., Smorenburg, C.H., Korevaar, J.C., Siesling, S. Patient-reported health problems and healthcare use after treatment for early-stage breast cancer The Breast: 2019, 46, 4-11

(57\%), and axillary dissection (21\%). Ninety-three percent experienced one or more health problems.

Over $50 \%$ of respondents experienced fatigue, psychological problems, and health problems regarding

the breast, and/or musculoskeletal, central nervous, and reproductive system. Treatment with chemotherapy

was significantly associated $(p<0.05)$ with an increased risk of health problems, respectively

fatigue (OR:2.00), respiratory (OR:1.81), gastrointestinal (OR:1.87), central nervous (OR:3.40), and skin

problems (OR:2.62). Use of healthcare for one or more health problems was reported by $64 \%$ of respondents.

Discussion: Almost all patients experienced health problems up to five years after breast cancer diagnosis,

with a range of complaints that were consistently present over time. Factors associated with the

development of health problems are useful for better informing patients beforehand and targeting

follow-up care.

\section{Introduction}

Breast cancer and its treatment have a major impact on psychosocial, emotional, cognitive, and physical well-being [1e3], partly due to treatment-induced effects later. Surgery (including axillary resection) is associated with lymphoedema, pain in the arm and shoulder, and movement restrictions of the arm and shoulder [4]. Radiotherapy can lead to pneumonitis, skin changes, and pain and lymph oedema in the arm following nodal irradiation [4,5]. Chemotherapy can lead to premature menopause, neuropathy [4] and bone loss [5]. Both chemotherapy and anti-hormonal therapy were also negatively associated with cognitive decline, although the evidence is not conclusive $[4,6]$. Cardiotoxic effects can follow radiotherapy, chemotherapy (anthracyclines) and targeted therapies (anti-HER2 treatment and trastuzumab) $[4,5,7,8]$. Other common effects of breast cancer diagnosis and treatment were fatigue, insomnia, depression and cognitive dysfunction $[1,4,6]$, and one third of patients experienced sexual problems, which were more pronounced in patients who were treated with chemotherapy [4]. All in all, the transition from treatment to 'life after cancer' is a difficult one, and can result in unmet needs [9]. Patients receive follow-up care to inform and counsel them about these potential physical and psychosocial late effects of the treatment and to detect these effects at an early stage [10e12]. International guidelines prescribe that follow-up visits should take place several times per year up to five years after completing treatment [10e12]. These visits are made in a hospital care setting, are often related to monitoring for recurrences of breast cancer and are done by a physician or nurse specialising in breast cancer care [10]. Although knowledge about (late) treatmentrelated effects is available for each treatment modality, several studies concluded that providing information to patients during follow-up could be improved: patients were often unaware of what effects to expect later and the duration of these effects, and thus felt unprepared for the posttreatment period $[3,13 \mathrm{e} 15]$. Patients also have expressed strong unmet needs for education, information, or support related to physical impairments or activity limitations [3]. A complete overview of risks of developing health problems after each of the breast cancer treatment modalities is useful in anticipating the informational and other needs of patients during the follow-up period. The primary goal of this study was to identify treatmentrelated health problems in breast cancer patients 
Ligt, K.M. de, Heins, M., Verloop, J., Smorenburg, C.H., Korevaar, J.C., Siesling, S. Patient-reported health problems and healthcare use after treatment for early-stage breast cancer The Breast: 2019, 46, 4-11

up to five years after completing curative treatment. A secondary aim was to identify use of care associated with these health problems.

\section{Materials and methods}

Twenty hospitals in the Netherlands (8 general hospitals, 10 teaching hospitals, and 2 academic hospitals) were willing to take part by inviting patients to fill in an online survey. In each participating hospital, fifty patients were selected from the Netherlands Cancer Registry (NCR). The NCR had documented data about incidence, diagnosis, and treatment since 1989 for all cancer patients treated in Dutch hospitals [16]. Those selected for inclusion were surgically-treated female breast cancer patients, diagnosed with early stage disease (stages I to III), aged 18 and older and diagnosed between 2012 and 2016. Patients were excluded if they were undergoing treatment for recurrent disease, could not read or write Dutch, were not receiving active follow-up anymore, or if their contact information was not up to date (n $1 / 4124)$. Paper questionnaires were provided on request for patients without computer access.

From September 2017 to March 2018, 876 patients were invited by letter through the patient administration systems of each participating hospital; responses were collected until May 2018. Each letter contained individual log in credentials for the PROFILES Registry ('Patient Reported Outcomes Following Initial treatment and Long-term Evaluation of Survivorship'), an online survey application developed to study the physical and psychosocial impact of cancer and its treatment from a cohort of cancer survivors [17]. On first using this application, patients set up their own password and give consent for their responses to be processed and merged with their clinical data in the NCR. The Dutch Medical Research (Human Subjects) Act does not apply for this type of study and formal approval from an ethics committee was not required. The use of NCR data in this study was approved by the NCR Privacy Review Board.

The self-administered survey (Appendix 1) consisted of three topics: 1) a predefined list of health problems experienced during the twelve months preceding the questionnaire, retrieved from Yzermans et al. [18]; 2) associated use of health care (visiting a physician or general practitioner) over the last year; 3) personal information including highest completed level of education, current treatment status, and comorbidities. Comorbidity was defined as chronic health problems that patients experienced at the time of completing the survey, including other types of cancer, pulmonary, cardiovascular, gastrointestinal, urogenital, musculoskeletal, neurological, metabolic/coagulation, or infectious diseases. The questionnaire was tested by a panel of current and former breast cancer patients for its readability and comprehensibility.

\subsection{Analyses}

The patient-reported data from the questionnaire was linked to the clinical data in the NCR. The following analyses were performed. First, respondent characteristics (age, year of diagnosis, type of surgery, stage of disease, and type of hospital) were compared against non-respondent characteristics to assess generalisability (chi-square, level of significance $p<0.05$ ). Second, patient characteristics and patient-reported health problems plus health care use were presented. Health problems were categorised according to organ system, resulting in eleven categories, and presented against time since diagnosis. Thirdly, the effect of patient and treatment characteristics on the health problems experienced was tested through multivariate logistic regression analyses. A regressionwas formed for each category of health problems. Levels of significance of 0.10 and 0.05 were applied for univariate and multivariate regression, respectively. Factors tested in relation to reported health problems were: age at time of the survey, time since diagnosis (in years), highest completed level of education, presence of comorbidities, lateralisation of disease, type of surgical intervention (whether or not combined with radiotherapy), axillary surgery, chemotherapy (whether or not combined with targeted 
Ligt, K.M. de, Heins, M., Verloop, J., Smorenburg, C.H., Korevaar, J.C., Siesling, S. Patient-reported health problems and healthcare use after treatment for early-stage breast cancer The Breast: 2019, 46, 4-11

therapy), anti-hormonal therapy, and breast reconstruction surgery. All analyses were performed in STATA SE14.2 [19].

\section{Results}

Out of 876 invited patients, 404 completed the questionnaire (46\%). Respondents were a representative sample of the invited population based on patient and treatment characteristics, with a slight underrepresentation for the youngest and oldest patients ( $20 \%$ for respondents versus $25 \%$ for non-respondents for age<50; $17 \%$ for respondents versus $23 \%$ for non-respondents ages $70 p ; p 1 / 4$ 0.010 , results not shown). Table 1 shows all patient characteristics. The median age at the time of response was 62.0 years (range: $27.5 e 91.6)$. One (33\%) or more than one (15\%) comorbid diseases were present in $48 \%$ of patients. Of all patients, $59 \%$ received breast conserving therapy and $41 \%$ received a mastectomy; $21 \%$ also received axillary dissection, and $72 \%$ received treatment with radiotherapy. Adjuvant or neoadjuvant treatment with chemotherapy and anti-hormonal therapy (categories not mutually exclusive) was given to $49 \%$ and $57 \%$ respectively; chemotherapy was combined with trastuzumab in one quarter of patients treated with chemotherapy. These treatment characteristics are representative for the surgically treated breast cancer population in the Netherlands.

\subsection{Health problems}

Health problems were grouped in eleven categories based on organ system (Table 2). In total, 377 out of 404 (93\%; Fig. 1) respondents experienced one or more health problems in at least one of these categories. Patients most often reported complaints in the following categories: musculoskeletal health problems (70.5\%); central nervous system health problems (66.1\%), in particular memory and concentration impairment (42.6\%); fatigue (63.4\%), reproductive system health problems (54.5\%), problems related to the breast (54.0\%) and psychological health problems (53.0\%; Fig. 1).

When stratified by time since diagnosis, the majority of health problems were present to the same extent over time (Table 3). Only the number of patients with fatigue (78\%e56\%, p $1 / 40.033$ ), hair loss (34\%e15\%, p 1/4 0.023) and premature menopause (53\%e35\%, p 1/4 0.024) decreased significantly over time, whereas the number of patients with pain and/or complaints in the back (19\%e44\%, p $1 / 40.022)$ and pain and/or complaints in the lower extremities (27\%e51\%, p $1 / 40.023)$ increased significantly over time.

\subsection{Effect of patient and treatment characteristics on experiencing health problems}

Surgical treatment with adjuvant radiotherapy was provided in $58 \%$ and $14 \%$ of patients with breast conserving surgery and amputation respectively; $25 \%$ had amputations without radiotherapy. At the time of the survey, $39 \%$ of patients were still being treated with anti-hormonal therapy, $11 \%$ had finished treatment ( $7 \%$ unknown (n $1 / 428)$ ). Chemotherapy was administered in $50 \%$ of patients, of which one quarter received additional trastuzumab. Treatment modalities that were significantly associated with reported health problems based on the multivariate analyses are shown in Table 4.

Surgery with adjuvant radiotherapy treatment and axillary dissection were significantly associated with a higher risk of problems in the breast area (breast conserving surgery: OR:3.16, $p<0.001$; amputation: OR:2.25, p 1/4 0.032; axillary dissection: OR:2.84, p 1/4 0.001). Immediate breast reconstruction was associated with musculoskeletal health problems (OR:4.44, p 1/4 0.022). Treatment with chemotherapy was significantly associated with an increased risk for fatigue (OR: 2.00, p $1 / 40.020$, with trastuzumab OR: 2.40, p 1/4 0.031), respiratory health problems (OR:1.81, p 1/4 0.029; with trastuzumab OR:2.74, p 1/4 0.005), gastrointestinal health problems (OR:1.87, p 1/4 0.011; with trastuzumab OR:2.06, p 1/4 0.035), central nervous system health problems (OR:3.40, $p<0.001$; with trastuzumab OR:2.51, p 1/4 0.029), and skin health problems (OR:2.62, p < 0.001; with trastuzumab 
Ligt, K.M. de, Heins, M., Verloop, J., Smorenburg, C.H., Korevaar, J.C., Siesling, S. Patient-reported health problems and healthcare use after treatment for early-stage breast cancer The Breast: 2019, 46, 4-11

OR:2.51, p 1/4 0.029). Currently receiving anti-hormonal therapy was significantly associated with an increased risk of fatigue (OR:1.73, p 1/4 0.040), and reproductive system health problems (OR:1.91, p $1 / 40.019)$. Completed administration of anti-hormonal therapy was significantly associated with a reduction in central nervous system health problems (OR:0.42, p 1/4 0.028). Supplementary Table 1 shows factors that were significant in univariate and multivariate testing. The area under the ROC curve for all eleven regression models ranged between 0.6438 (for skin problems) and 0.7791 (for reproductive system health problems).

\subsection{Use of care}

Of the $93 \%$ percent of respondents who experienced health problems, $64 \%$ used care for at least one of those problems (Fig. 1). Patients most often visited their physician for fractures (78.6\%), gastric/abdominal complaints (51.0\%), and skin problems (50.0\%), and least often for infertility $(0.0 \%)$, dry mouth (4.7\%), and hypersensitivity to light or sound (6.1\%). The most commonly reported health problems such as fatigue (63.4\%), memory/concentration problems (42.6\%), and menopausal complaints (39.1\%) were not necessarily the ones for which the most use of care was reported $(27.3 \%$, $8.1 \%$, and $12.0 \%$ respectively).

\section{Discussion}

In this study, which uses a survey from 404 women previously treated for early-stage breast cancer, $93 \%$ of respondents experienced one or more health problems during the five-year period after diagnosis. The most frequently reported categories of health problem (by $>50 \%$ of respondents) were: musculoskeletal or related to the nervous system, fatigue, reproductive system, breast area, and psychological health. The majority of health problems were reported consistently over time. The present study found that treatment with chemotherapy and anti-hormonal therapy in particular were associated with a range of health problems. A secondary aim of this studywas to identify use of care for the health problems reported. The three most commonly reported health problems for which care was used were fractures, gastric/abdominal complaints, and skin problems. Care was used least for infertility, dry mouth, and hypersensitivity to light or sound.

The results of the present study fit with the literature about treatment-induced effects in breast cancer patients. In multivariate regression analyses, treatment with chemotherapy and antihormonal therapy in particular were associated with an increase in several health problems. The literature has already described a wide range of complaints resulting from chemotherapy, including premature menopause, pneumonitis, bone loss, and skin changes, cardiotoxic effects, neuropathy, and cognitive decline [4e6]. The present study found a positive association between receiving antihormonal therapy and reproductive system health problems. Literature states that anti-hormonal treatment may lead to vasomotor symptoms, sexual dysfunction, and infertility [4]. In total, $17 \%$ of our respondents reported problems with sexuality. The actual proportion of patients with sexuality problems may be higher, as patients may feel uncomfortable reporting these problems [20]. Furthermore, the negative effects of autologous breast reconstruction on arm symptoms, and breast conserving therapy (with additional radiotherapy) on breast symptoms have already been described in the literature [21]. The present study found an association between breast reconstruction and musculoskeletal problems (including arm symptoms), and between radiotherapy treatment and health problems in the breast (including skin problems). However, in the utilised database, only data about immediate breast reconstruction was available in the database used, whereas patients treated with radiotherapy may have received delayed breast reconstruction to avoid these effects [22]. Almost all health problems were reported consistently over the time since diagnosis (except for fatigue, hair loss and menopausal complaints), suggesting the majority of problems remained present over time. This was confirmed in a cohort study by Van Leeuwen et al. [2]. The decrease in menopausal complaints may follow from 
Ligt, K.M. de, Heins, M., Verloop, J., Smorenburg, C.H., Korevaar, J.C., Siesling, S. Patient-reported health problems and healthcare use after treatment for early-stage breast cancer The Breast: 2019, 46, 4-11

decreasing therapy adherence to anti-hormonal treatment [23]; Hershman et al. [24] stated that 18\% of patients are non-compliant in the first two years. This underlines the need for longitudinal studies to gain an understanding of the possible ongoing cancerrelated late effects, that may not have the same development over time in patients. This is important, because persistent symptoms or sideeffects have been associated with distress and poor quality of life for breast cancer survivors and cancer survivors in general $[9,13]$. This negative effect of continuing symptoms on the quality of life is important regarding the use of care that was evaluated in the present study, which also sheds light on the share of health problems that is presented to the physician: almost all breast cancer patients experienced health problems, but only half of the respondents reported the use of care for one or more of these. Lubberding et al. [15] described how post-treatment symptoms often remain unknown to health care providers, for both social (not wanting to bother people in their surroundings) and practical reasons (short consultation time, not having a follow-up appointment when symptoms emerged, not knowing who to contact in the event of symptoms). However, they noted as well that a complete overview of the patient's health status was missing, resulting in a lack of coordination of care and suboptimal referral to supporting care services. However, a complete overview of health status should be the foundation of follow-up for later effects of breast cancer treatment. During consultations, this overview could be obtained by administering and discussing of patient-reported outcome measures that consist of symptom-specific measurement scales addressing both physical and psychosocial topics $[25,26]$, or by symptom checklists $[27,28]$. Long-term regular assessment of these measures during follow-up means that health problems that persist become a burden later are detected as well. Discrepancies with the literature were found as well. No treatment factors were associated with the risk of cardiac health problems, even though the risk of cardiovascular disease is increased by $30 \%$ in cancer survivors and was significantly associated with radiotherapy and systemic treatment $[7,8,29]$. The increased risks for coronary events starts within the five years after adiotherapy, but may continue for up to $20 \mathrm{e} 30$ years $[8,29]$. Effects that develop over a period of time longer than five years, may therefore have been missed in the present study. Furthermore, no significant association between chemotherapy and reproductive system health problems was found, whereas literature reports infertility in more than half of patients younger than 40 [4]. However, only $4 \%$ of respondents (n $1 / 416$ ) were younger than 40 , possibly reducing the power to detect this effect. Some notes are needed when interpreting the results of this study. The application of patient-reported surveys is related to forms of bias, including recall bias and response bias. Recall bias might lead to underestimation of complaints, as patients may not recall all the health problems they experienced. Response bias may lead to underestimation or overestimation, as patients with poorer health may either feel more urgency or feel too sick to participate. Underestimation of health problems may have occurred, as $79 \%$ of patients with fractures reported use of the healthcare system, where this would be expected to be $100 \%$. The interpretation of our results could be improved by comparing them against health problems in the general population, to better understand which health problems are more prevalent in breast cancer patients. This step will be undertaken in our further research. Most breast cancer patients (76\%) in a study by Hagen et al. [30] reported health complaints equally as often as the controls, however, health problems such as leg pain, interrupted sleep, hot flushes, tiredness, dizziness, and diarrhoea were reported significantly more by patients than controls. Lastly, interaction between treatment modalities that were associated with the health problems reported were tested, but no interactions were found. Friese et al. [31] found that receiving combined chemotherapy and radiation therapy was associated with a greater severity of toxicity reported. 4.1. Implications for practice Breast cancer is the most commonly diagnosed cancer in women worldwide, estimated at 2.1 million new diagnoses in 2018 [32]. Breast cancer patients in North America and Europe have a five-year survival of over $80 \%$ [33]. The extrapolation of the health problems found in the present study to this enormous group of surviving patients endorses the importance of proper follow-up care. The factors associated with the health problems found in 
Ligt, K.M. de, Heins, M., Verloop, J., Smorenburg, C.H., Korevaar, J.C., Siesling, S. Patient-reported health problems and healthcare use after treatment for early-stage breast cancer The Breast: 2019, 46, 4-11

this study could be used in multiple ways. First, they could be helpful in recognising current symptoms in relation to previous cancer treatment [9]. This could also be important for general practitioners, who might be consulted by patients about health issues induced by breast cancer treatment long after follow-up is completed [9]. Secondly, it would be applicable in a prospective model of monitoring, to promote monitoring and healthy behaviours, to provide education about health problems and early identification of them, and to introduce rehabilitation when limitations are identified [34]. Thirdly, information about health problems after breast cancer treatment focus more on specific target groups, ranging from patients with low to high burdens of symptoms in terms of pain, psychological complaints and fatigue, and burdens due to other complaints [35]. Patient-reported outcome measures (PROMS), for instance the standard set suggested by the International Consortium for Health Outcomes Measurement (ICHOM) Initiative, can be used to monitor health problems and quality of life [36].

\section{Conclusion}

Almost all patients treated for early-stage breast cancer experienced health problems up to five years after diagnosis. The most frequent categories of health problems (>50\%) were related to the musculoskeletal system, nervous system, fatigue, reproductive system, breast area, and psychological health. Treatment with chemotherapy and anti-hormonal therapy in particular were associated with health problems being reported. Factors associated with the development of health problems are useful for informing patients better beforehand and for targeting follow-up care.

\section{Conflicts of interest}

None.

\section{Ethical approval}

The Medical Research (Human Subjects) Act does not apply for this type of study and formal approval from an ethics committee was not required.

\section{Acknowledgements}

The authors would like to thank all the women who participated in this study, who were kindly invited through all the participating hospitals: Beatrix Ziekenhuis, Bernhoven Ziekenhuis, Canisius Wilhelmina Ziekenhuis, Diakonessenhuis, Erasmus MC, Gelre Ziekenhuizen, IJsselland Ziekenhuis, Lange Land Ziekenhuis, Maxima Medisch Centrum, Medisch Centrum Haaglanden, Medisch Spectrum Twente, NoordWest Ziekenhuisgroep, Rijnstate Ziekenhuis, Sint Jansdal, St Deventer Ziekenhuis, Streekziekenhuis Koningin Beatrix, ZiekenhuisGroep Twente, and UMC St Radboud. Also many thanks for her efforts to our research intern Ms Beldhuis.

\section{Appendix A. Supplementary data}

Supplementary data to this article can be found online at https://doi.org/10.1016/j.breast.2019.03.010.

\section{References}

[1] Kenyon M, Mayer DK, Owens AK. Late and long-term effects of breast cancer treatment and surveillance management for the general practitioner. J Obstet Gynecol Neonatal Nurs

2014;43:382e98. 
Ligt, K.M. de, Heins, M., Verloop, J., Smorenburg, C.H., Korevaar, J.C., Siesling, S. Patient-reported health problems and healthcare use after treatment for early-stage breast cancer The Breast: 2019, 46, 4-11

[2] van Leeuwen $M$, et al. Understanding the quality of life (QOL) issues in survivors of cancer: towards the development of an EORTC QOL cancer survivorship questionnaire. Health Qual Life Outcomes 2018;16(1).

[3] Binkley JM, et al. Patient perspectives on breast cancer treatment side effects and the prospective surveillance model for physical rehabilitation for women with breast cancer. Cancer 2012;118:2207e16.

[4] Ewertz M, Jensen AB. Late effects of breast cancer treatment and potentials for rehabilitation. Acta Oncol 2011;50:187e93.

[5] Agrawal S. Late effects of cancer treatment in breast cancer survivors. South Asian J Canc 2014;3(2).

[6] Pinto AC, de Azambujab E. Improving quality of life after breast cancer: dealing with symptoms. Maturitas 2011;70:343e8.

[7] Eschenhagen T, et al. Cardiovascular side effects of cancer therapies: a position statement from the Heart Failure Association of the European Society of Cardiology. Eur J Heart Fail 2011;13:1e10.

[8] Maas AHEM, et al. Cardiovascular surveillance in breast cancer treatment: a more individualized approach is needed. Maturitas 2016;89:58e62.

[9] Mayer DK, Nasso SF, Earp JA. Defining cancer survivors, their needs, and perspectives on survivorship health care in the USA. Lancet 2017;18(1): e11e8.

[10] IKNL. National guideline on breast cancer. Netherlands Comprehensive Cancer Organisation (IKNL); 2012.

[11] Runowicz CD, et al. American cancer society/American society of clinical oncology breast cancer survivorship care guideline. J Clin Oncol 2016;34(6): 611e35.

[12] Senkus E, et al. Primary breast cancer: ESMO Clinical Practice Guidelines for diagnosis, treatment and follow-up. Ann Oncol 2015;26(suppl_5):v8e30.

[13] Rosedale M, Fu MR. Confronting the unexpected: temporal, situational, and attributive dimensions of distressing symptom experience for breast cancer survivors. Oncol Nurs Forum 2010;37(1).

[14] Chawla N, et al. Quality of patient-provider communication among cancer survivors: findings from a nationally representative sample. J Oncol Pract 2016;12(12).

[15] Lubberding S, et al. Improving access to supportive cancer care through an eHealth application: a qualitative needs assessment among cancer survivors. J Clin Nurs 2015;24:1367e79.

[16] Netherlands Comprehensive Cancer Organisation (IKNL). Dutch cancer figures: about the registration [webpage]. 2018 [cited 2018 14-5-2018]; Available from:

https://www.cijfersoverkanker.nl/about-the-registration-37.html.

[17] van de Poll-Franse LV, et al. The Patient Reported Outcomes Following Initial treatment and Long term Evaluation of Survivorship registry: scope, rationale and design of an infrastructure for the study of physical and psychosocial outcomes in cancer survivorship cohorts. Eur J Cancer 2011;47(14):2188e94.

[18] Yzermans J, et al. Assessing non-specific symptoms in epidemiological studies: development and validation of the symptoms and perceptions (SaP) questionnaire. Int J Hyg Environ Health 2016;219(1):53e65.

[19] StataCorp. In: College Station TSL, editor. Stata statistical software: release, vol. 14; 2015.

[20] Stabile C, et al. Sexual health needs and educational intervention preferences for women with cancer. Breast Canc Res Treat 2017;165(1):77e84.

[21] Lagendijk M, et al. Patient reported outcome measures in breast cancer patients. Eur J Surg Oncol 2018;44:963e8.

[22] Mureau MAM, Nederlandse Vereniging voor Plastische Chirurgie (NVPC). Dutch breast reconstruction guideline. J Plast Reconstr Aes 2018:290e304. 
Ligt, K.M. de, Heins, M., Verloop, J., Smorenburg, C.H., Korevaar, J.C., Siesling, S. Patient-reported health problems and healthcare use after treatment for early-stage breast cancer The Breast: 2019, 46, 4-11

[23] Bluethmann SM, et al. Deconstructing decisions to initiate, maintain, or discontinue adjuvant endocrine therapy in breast cancer survivors: a mixedmethods study. Oncol Nurs Forum 2017;44(3). E101ee110.

[24] Hershman DL, et al. Psychosocial factors related to non-persistence with adjuvant endocrine therapy among women with breast cancer: the Breast Cancer Quality of Care Study (BQUAL). Breast Canc Res Treat 2016;157(1): 133e43.

[25] Wintner LM, et al. Quality of life research within the EORTCdthe EORTC QLQC30. Eur J Cancer 2016;68:73e81.

[26] Cano SJ, et al. A closer look at the BREAST-Q. Clin Plast Surg 2013;40:287e96.

[27] IKNL. Guideline on detecting psychosocial care needs in cancer patients. Netherlands Comprehensive Cancer Organisation (IKNL); 2017.

[28] Williams PD, et al. Therapy-related symptom checklist use during treatments at a cancer center. Cancer Nurs 2013;36(3):245e54.

[29] Naaktgeboren WR, et al. Long-term cardiovascular health in adult cancer survivors. Maturitas 2017;105:37e45.

[30] Hagen KB, et al. Fatigue, anxiety and depression overrule the role of oncological treatment in predicting self-reported health complaints in women with breast cancer compared to healthy controls. Breast 2016;28:100e6.

[31] Friese CR, et al. Treatment-associated toxicities reported by patients with early-stage invasive breast cancer. Cancer 2017;123(11):1925e34.

[32] Bray F, et al. Global cancer statistics 2018: GLOBOCAN estimates of incidence and mortality worldwide for 36 cancers in 185 countries. CA Cancer J Clin 2018;0:1e31.

[33] Coleman MP, et al. Cancer survival in five continents: a worldwide population-based study (CONCORD). Lancet Oncol 2008;9:730e56.

[34] Stout NL, et al. A prospective surveillance model for rehabilitation for women with breast cancer. Cancer 2012;118(8):2191e200.

[35] Avis NE, et al. Longitudinal examination of symptom profiles among breast cancer survivors. J Pain Symptom Manag 2017;53(4):703e10.

[36] Ong WL, et al. A standard Set of value-based patient-centered Outcomes for breast cancer: the international Consortium for health outcomes measurement (ICHOM) initiative. JAMA Oncol 2017;3(5):677e85. 
Ligt, K.M. de, Heins, M., Verloop, J., Smorenburg, C.H., Korevaar, J.C., Siesling, S. Patient-reported health problems and healthcare use after treatment for early-stage breast cancer The Breast: 2019, 46, 4-11

\section{Table 1}

Table 1

Respondent characteristics $(n=404)$.

\begin{tabular}{|c|c|c|}
\hline & $N(404)$ & $\%$ \\
\hline \multicolumn{3}{|l|}{ Patient characteristics } \\
\hline Mean (SD, range) & \multicolumn{2}{|c|}{$62.0(10.9,27.5-91.6)$} \\
\hline$<50$ & 56 & 14 \\
\hline $50-59$ & 108 & 27 \\
\hline $60-69$ & 133 & 33 \\
\hline $70+$ & 107 & 26 \\
\hline \multicolumn{3}{|l|}{ Time between diagnosis and survey } \\
\hline$<2$ & 83 & 21 \\
\hline $2-3$ & 92 & 23 \\
\hline $3-4$ & 85 & 21 \\
\hline $4-5$ & 89 & 22 \\
\hline$>5$ & 55 & 14 \\
\hline \multicolumn{3}{|l|}{ Highest completed level of education ${ }^{\mathrm{b}}$} \\
\hline Secondary education or lower & 122 & 30 \\
\hline Medium vocational training (MBO) & 170 & 42 \\
\hline Higher vocational training (HBO/university) & 108 & 27 \\
\hline \multicolumn{3}{|l|}{ Comorbidity ${ }^{\mathrm{a}, \mathrm{b}}$} \\
\hline None & 188 & 47 \\
\hline One & 131 & 33 \\
\hline Two or more & 61 & 15 \\
\hline \multicolumn{3}{|l|}{ Tumour characteristics } \\
\hline \multicolumn{3}{|l|}{ Year of diagnosis } \\
\hline 2012 & 54 & 13 \\
\hline 2013 & 92 & 23 \\
\hline 2014 & 86 & 21 \\
\hline 2015 & 89 & 22 \\
\hline 2016 & 83 & 21 \\
\hline \multicolumn{3}{|l|}{ Stage } \\
\hline I & 186 & 46 \\
\hline II & 174 & 43 \\
\hline III & 44 & 11 \\
\hline \multicolumn{3}{|l|}{ Hormone receptor status ${ }^{\text {a }}$} \\
\hline HR-positive & 287 & 71 \\
\hline ER or PR positive & 53 & 13 \\
\hline HR-negative & 62 & 15 \\
\hline \multicolumn{3}{|l|}{ Tumour grade ${ }^{a}$} \\
\hline 1 & 95 & 24 \\
\hline 2 & 176 & 44 \\
\hline 3 & 95 & 24 \\
\hline \multicolumn{3}{|l|}{ Treatment characteristics } \\
\hline \multicolumn{3}{|l|}{ Surgery ${ }^{a}$} \\
\hline Breast conserving surgery & 238 & 59 \\
\hline Mastectomy & 160 & 41 \\
\hline \multicolumn{3}{|l|}{ Local treatment modalities: } \\
\hline Axillary dissection & 85 & 21 \\
\hline Radiotherapy & 291 & 72 \\
\hline Immediate breast reconstruction & 36 & 9 \\
\hline \multicolumn{3}{|l|}{ Systemic treatment modalities: } \\
\hline Chemotherapy & 196 & 49 \\
\hline - With trastuzumab & 50 & 12 \\
\hline Anti-hormonal therapy & 232 & 57 \\
\hline \multicolumn{3}{|l|}{ Hospital characteristics } \\
\hline \multicolumn{3}{|l|}{ Hospital type ${ }^{c}$} \\
\hline General hospital & 166 & 41 \\
\hline Teaching/academic hospital & 238 & 59 \\
\hline \multicolumn{3}{|l|}{ Hospital volume ${ }^{\mathrm{d}}$} \\
\hline Low & 157 & 39 \\
\hline Medium & 88 & 22 \\
\hline High & 159 & 39 \\
\hline
\end{tabular}

Totals do not add up due to missing values.

Patient-reported.

${ }^{c}$ Hospitals were categorised as either general, teaching, or academic hospitals.

d Number of surgically treated non-metastatic breast cancer patients per year (average over 2012-2016), categorised as low ( $<100)$, medium (100-149), and high $(>150)$ volume. 
Ligt, K.M. de, Heins, M., Verloop, J., Smorenburg, C.H., Korevaar, J.C., Siesling, S. Patient-reported health problems and healthcare use after treatment for early-stage breast cancer The Breast: 2019, 46, 4-11

\section{Table 2}

Table 2

Health problems categorised by organ system.

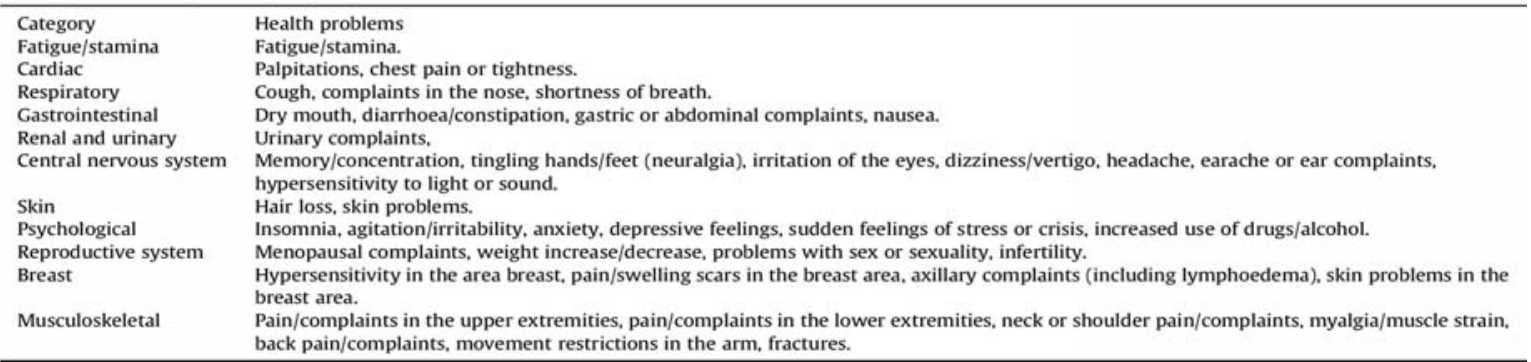

Table 3

Table 3

Patient-reported health problems presented per year of diagnosis.

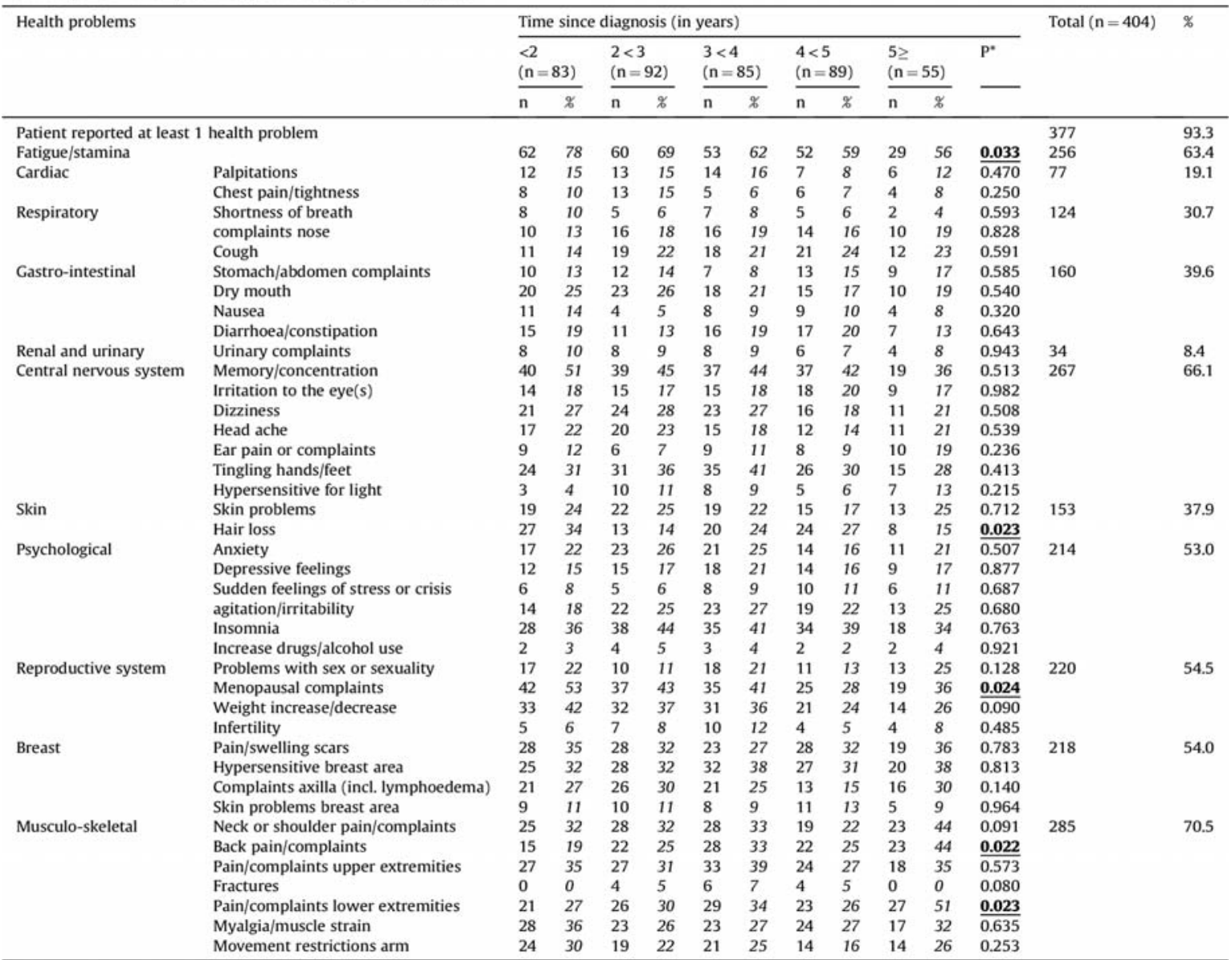

"Chi-squared tested (level of significance: 0.05 ). 
Ligt, K.M. de, Heins, M., Verloop, J., Smorenburg, C.H., Korevaar, J.C., Siesling, S. Patient-reported health problems and healthcare use after treatment for early-stage breast cancer The Breast: 2019, 46, 4-11

\section{Table 4}

Table 4

The effect of treatment factors on patient-reported health problems..

\begin{tabular}{|c|c|c|c|c|c|c|c|c|c|c|c|c|c|}
\hline \multirow{4}{*}{\begin{tabular}{|l}
$\begin{array}{r}\text { Treatment } \\
\text { modality' }\end{array}$ \\
Group size (\%) \\
Health problems':
\end{tabular}} & \multicolumn{3}{|c|}{ Surgical treatment and radiotherapy } & \multirow{2}{*}{\multicolumn{2}{|c|}{ Axillary dissection }} & \multirow{2}{*}{\multicolumn{2}{|c|}{ Breast reconstruction }} & \multicolumn{3}{|c|}{ Anti-hormonal therapy } & \multicolumn{3}{|c|}{ Chemotherapy } \\
\hline & \multirow{2}{*}{\begin{tabular}{|c|}
$\begin{array}{c}\text { BCS incl. } \\
\text { radiotherapy }\end{array}$ \\
$233(58)$ \\
\end{tabular}} & \multirow{2}{*}{\begin{tabular}{c|}
$\begin{array}{c}\text { AMP excl. } \\
\text { radiotherapy }\end{array}$ \\
$102(25)$ \\
\end{tabular}} & \multirow{2}{*}{\begin{tabular}{|c|}
$\begin{array}{c}\text { AMP incl. } \\
\text { radiotherapy }\end{array}$ \\
$58(14)$ \\
\end{tabular}} & & & & & \multirow{2}{*}{$\begin{array}{c}\begin{array}{c}\text { Not } \\
\text { applied }\end{array} \\
172(43) \\
\end{array}$} & \multirow{2}{*}{\begin{tabular}{|c|} 
ongoing \\
\\
$159(39)$ \\
\end{tabular}} & \multirow{2}{*}{\begin{tabular}{|c|} 
completed \\
$45(11)$ \\
\end{tabular}} & \multirow{2}{*}{\begin{tabular}{|c|}
$\begin{array}{c}\text { Not } \\
\text { applied }\end{array}$ \\
$208(51)$ \\
\end{tabular}} & \multirow{2}{*}{\begin{tabular}{|c|} 
Applied \\
$146(36)$ \\
\end{tabular}} & \multirow{2}{*}{\begin{tabular}{|c|c}
$\begin{array}{c}\text { Applied + } \\
\text { Trastuzumab }\end{array}$ \\
$50(12)$ \\
\end{tabular}} \\
\hline & & & & $319(79)$ & $85(21)$ & $368(91)$ & $36(9)$ & & & & & & \\
\hline & & & & & & & & & & & & & \\
\hline $\begin{array}{l}\text { Fatigue/ } \\
\text { stamina }\end{array}$ & - & ref & - & ref & - & ref & - & ref & 1.01 & $\begin{array}{c}1.73^{*} \\
(0.03-2.911 \\
\end{array}$ & ref & $\begin{array}{c}2.00^{*} \\
(1.12-3.57)\end{array}$ & $\begin{array}{c}2.40^{*} \\
(1.09-5.31)\end{array}$ \\
\hline Cardiac & - & ref & $\cdot$ & ref & - & ref & - & ref & $\cdot$ & - & ref & - & $\cdot$ \\
\hline Respiratory & - & ref & - & ref & - & ref & - & ref & - & - & ref & $\begin{array}{c}1.81^{*} \\
(1.06-3.08)\end{array}$ & $\begin{array}{c}2.74^{*} \\
(1.36-5.54) \\
\end{array}$ \\
\hline Gastrointestinal & - & ref & - & ref & - & ref & - & ref & - & - & ref & $\begin{array}{c}1.87^{*} \\
(1.15-3.01) \\
\end{array}$ & $\begin{array}{c}2.06^{*} \\
(1.05-4.04) \\
\end{array}$ \\
\hline Renal and urinary & - & ref & $\cdot$ & ref & - & ref & - & ref & - & - & ref & - & - \\
\hline $\begin{array}{c}\text { Central nervous } \\
\text { system }\end{array}$ & - & ref & - & ref & - & ref & - & ref & $\begin{array}{l}0.42^{*} \\
(0.19- \\
0.91) \\
\end{array}$ & 1.20 & ref & $\begin{array}{c}3.40^{*} \\
(1.80-6.42)\end{array} \mid$ & $\begin{array}{c}2.51^{*} \\
(1.10-5.74)\end{array}$ \\
\hline Skin & - & ref & - & ref & - & ref & - & ref & - & - & ref & \begin{tabular}{|c|}
$2.62^{*}$ \\
$(1.59-4.34)$ \\
\end{tabular} & $\begin{array}{c}2.13^{*} \\
(1.09-4.21) \\
\end{array}$ \\
\hline Psychological & . & ref & - & ref & 1.66 & ref & 1.21 & ref & $\cdot$ & - & ref & 1.66 & 1.21 \\
\hline $\begin{array}{l}\text { Reproductive } \\
\text { system }\end{array}$ & - & ref & - & ref & 1.13 & ref & - & ref & 0.60 & $\begin{array}{c}1.91^{*} \\
(1.11-3.27)\end{array}$ & ref & 1.73 & 2.07 \\
\hline Breast & $\begin{array}{c}3.16^{*} \\
(1.75-5.69) \\
\end{array}$ & ref & $\begin{array}{c}2.25^{*} \\
(1.07-4.72) \\
\end{array}$ & ref & $\begin{array}{c}2.84^{*} \\
(1.52-5.30) \\
\end{array}$ & ref & 0.87 & ref & - & - & ref & - & - \\
\hline Musculoskeletal & - & ref & - & ref & 1.66 & ref & $\begin{array}{c}4.44^{*} \\
(1.24-15.85) \\
\end{array}$ & ref & 0.77 & 1.49 & ref & 1.49 & 0.96 \\
\hline \multicolumn{14}{|c|}{ CS: breast conserving surgery: AMP: amputation } \\
\hline \multicolumn{14}{|c|}{$\begin{array}{l}\text { Factors that were significant in univariate testing }(p<0.10) \text { were included in multivariate testing. Cells are empty when factors were not significant in univariate testing and thus not included in } \\
\text { multivariate testing. Reference categories were patients treated without the specified treatment modalities. } \\
\text { Corrected for age at time of the survey, years since diagnosis, highest completed level of education, presence of comorbidities, and lateralisation of disease. }\end{array}$} \\
\hline
\end{tabular}


Ligt, K.M. de, Heins, M., Verloop, J., Smorenburg, C.H., Korevaar, J.C., Siesling, S. Patient-reported health problems and healthcare use after treatment for early-stage breast cancer The Breast: 2019, 46, 4-11

Fig. 1. Patient-reported health problems and use of care.

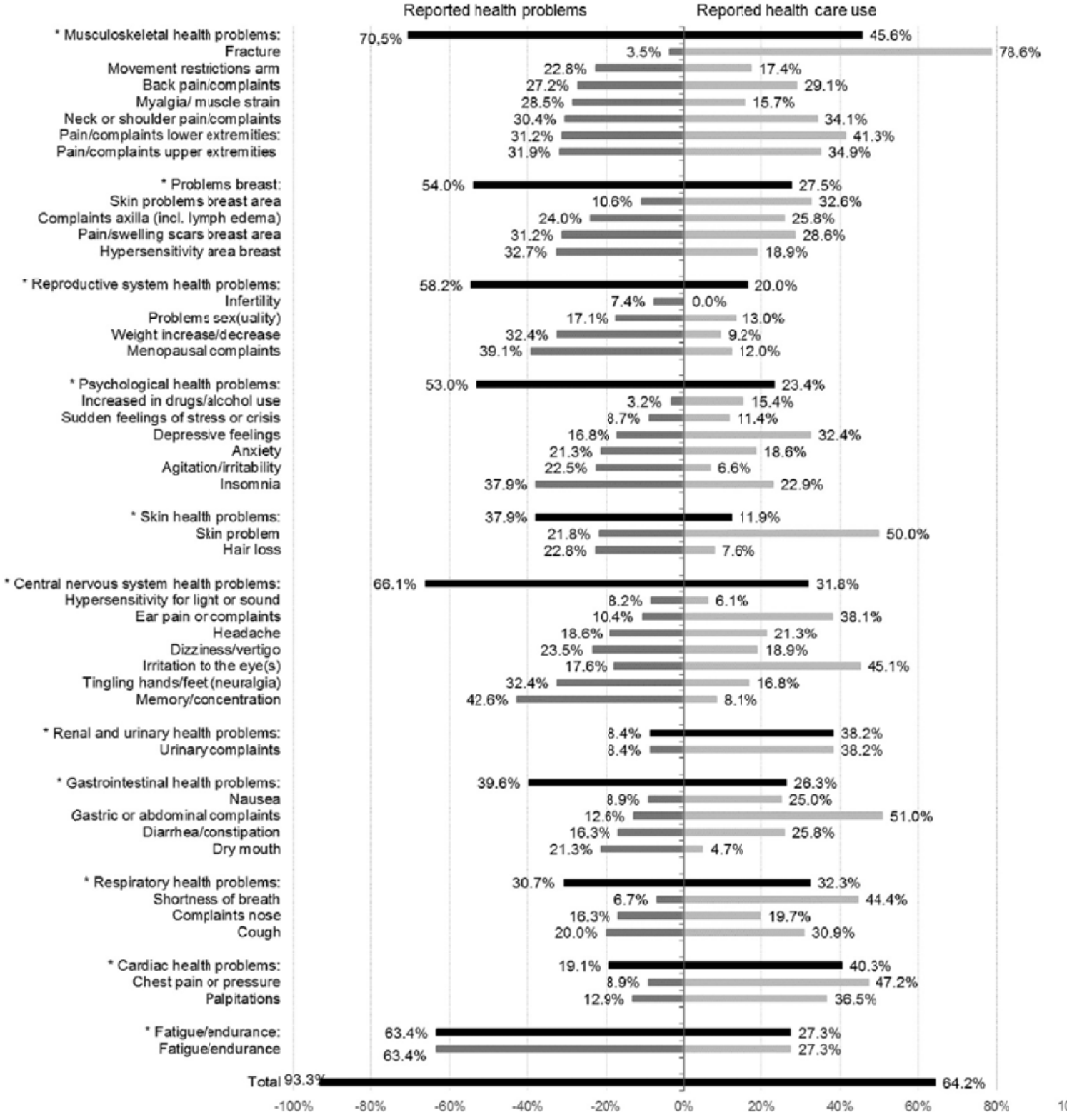

